\title{
Bald impfen gegen Rauchen und Bluthochdruck?
}

Chefredakteur Dr. med. Dirk Einecke
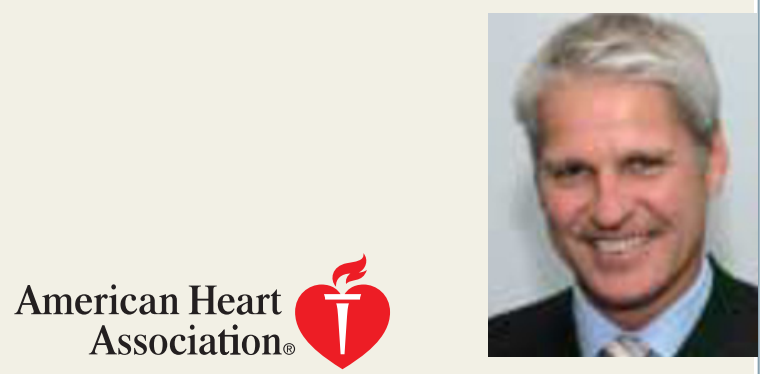

\section{BLUTHOCHDRUCK}

\section{Impfung im klinischen Test}

Ob sie die medikamentöse Behandlung des Bluthochdrucks ersetzen oder lediglich unterstützen kann, ist noch unklar. Tatsache ist: Schweizer Wissenschaftler haben eine Impfung gegen Hypertonie entwickelt. Der Impfstoff besteht aus viralen Partikeln, an die Angiotensin II gekoppelt wurde. Das Immunsystem von Impflingen fällt dann über Angiotensin II

\section{THERAPIE DES BLUTHOCHDRUCKS}

\section{Im Winter klappt sie schlechter}

In den kommenden Monaten sollten Sie Ihre Hochdruckpatienten aggressiver behandeln als sonst. Denn wie Wissenschaftler aus Washington herausfanden, ist eine antihypertensive Therapie im Winter seltener von Erfolg gekrönt als im Sommer - unabhängig davon, ob der

\section{BELASTUNGS-EKG}

\section{Armübungen tun es auch}

Wenn alte Menschen arthrosebedingt auf dem Ergometer nicht mehr so recht auf Touren kommen, Sie aber dringend ein Belastungs-EKG benötigen, kann der Test auch mit einem Arm-Ergometer durchgeführt werden, berichten Autoren aus St. Louis. Die Ergebnisse der körperlichen Übung nur mit den oberen Extremitäten sagten in einer großen Studie mit hoher Zuverlässigkeit die kardiovaskuläre Prognose voraus. her, einen der treibenden Faktoren des Bluthochdrucks.

In einer Testreihe wurden 72 Hypertoniker geimpft. Sie reagierten mit dosisabhängiger Antikörperbildung, der Blutdruck sank im Schnitt um 5,6/2,8 mmHg. Erstaunlich der Effekt in der Gruppe mit der höchsten Dosis: Der Morgenblutdruck von 5 bis 8 Uhr sank um 25/13 $\mathrm{mmHg}$.
Patient in Alaska oder in Florida lebt. Der Unterschied ist signifikant. Als Ursache vermutet man den Winterspeck: In der kalten Jahreszeit bewegen sich die Patienten weniger und schlagen Weihnachten diätetisch über die Stränge.

\section{HERZINFARKT-RISIKO}

\section{Erhöht bei Auto- und Fernseh-Besitzern}

Welcher ältere Mensch hat schon kein Auto und keinen Fernseher? In Schweden scheint es solche Leute noch zu geben. Nun haben Wissenschaftler herausgefunden, dass diese Personen ein deutlich geringeres Herzinfarkt-Risiko haben als solche, die Zeit im Auto oder vor dem Fernseher sitzend verbringen, anstatt sich körperlich zu bewegen. Das Auto war dabei noch gefährlicher als der Fernseher.

\section{Zitat der Woche}

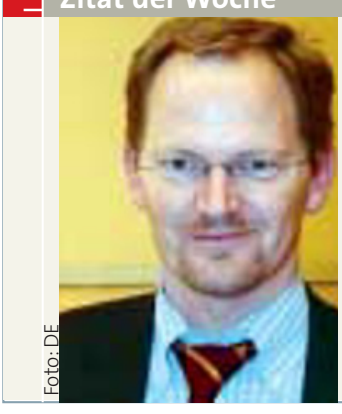

Wie misst man den Bauchumfang? „In der Mitte zwischen dem unteren Rippenbogen und dem Beckenkamm. Wichtig ist, dass man zwei Minuten lang misst, denn so lange kann kein Patient die Luft anhalten."

Prof. Dr. med. Andreas Hamann, Diabetes-Klinik Bad Nauheim, bei der Herbsttagung der Deutschen Gesellschaft für Diabetologie
NIKOTINABHÄNGIGKEIT

\section{Auch dagegen kann man impfen}

Ein Impfstoff, der die Bildung von nikotinspezifischen Antikörpern anregt, erzielt signifikante Effekte in der Raucherentwöhnung. In einer ersten Studie mit 301 noch gesunden Rauchern war der Impfstoff nach einem halben Jahr zu 24,6\% erfolgreich - im Vergleich zu 13\% in der Kontrollgruppe. Der Impfstoff heißt NicVAX ${ }^{\circledR}$ und wurde von Nabi Biopharmaceuticals entwickelt.

ENERGIE-DRINKS À LA RED BULL

\section{Hebt die Leistung - und den Blutdruck}

Wer täglich zwei koffein- und taurinhaltige Energiegetränke zu sich nimmt, steigert unter Ruhebedingungen seine Herzfrequenz um 5-7 Schläge pro Minute und seinen systolischen Blutdruck um $10 \mathrm{mmHg}$, berichten Forscher aus Detroit auf der Jahrestagung der „American Heart Association“. Für junge Herzgesunde mag das klinisch ohne Relevanz sein. Für Herzkranke und Hypertoniker kann dies aber gefährlich werden.

\section{MAHLZEITEN AUSLASSEN}

\section{Herzinfarktrisiko sinkt um $39 \%$}

Wer gelegentlich einen Tag lang alle Mahlzeiten weglässt, senkt eventuell sein Herzinfarktrisiko. Der 24-stündige Verzicht auf die üblichen Blutglukosebäder verschafft nicht nur den Gefäßwänden, sondern auch den pankreatischen Betazellen eine Verschnaufpause, vermutet B. Horne, University of Salt Lake City. Seine Arbeitsgruppe hatte beobachtet, dass Mormonen, die regelmäßig fasten, ein niedrigeres KHK-Risiko haben. Nun hat man festgestellt, dass Fasten auch aus nicht religiösen Gründen das KHKRisiko signifikant senkt - um 39\%. Die Beobachtung ist aber noch kein Beweis. 